\title{
RESTITUCIÓN DEL MENOR, DECLARACIÓN \\ DE ILICITUD DEL TRASLADO Y COMPETENCIA JUDICIAL \\ INTERNACIONAL. COMENTARIO AL AUTO \\ DE LA AUDIENCIA PROVINCIAL DE LAS ISLAS BALEARES \\ DE 19 DE ABRIL DE 2018
}

\author{
RETURN OF THE CHILD, DECLARATION OF WRONGFUL \\ REMOVAL AND JURISDICTION. COMMENT \\ ON THE JUDGMENT OF THE SPANISH COURT OF APPEAL \\ OF THE BALEARIC ISLANDS OF 19 APRIL 2018
}

\author{
Alberto Muñoz FernándeZ \\ Profesor Contratado Doctor \\ Universidad de Navarra
}

Recibido: 27.12.2018 / Aceptado: 21.01.2019

DOI: https://doi.org/10.20318/cdt.2019.4661

\begin{abstract}
Resumen: la solicitud de restitución de un niño ante las autoridades inglesas no impide que los tribunales españoles, país donde el menor tenía su de residencia habitual antes del traslado, declaren la ilicitud del traslado y adopten medidas cautelares para evitar un nuevo traslado internacional.

Palabras clave: sustracción internacional de menores, secuestro internacional de menores, restitución del menor, retorno inmediato, declaración de ilicitud del traslado, retirada del pasaporte, competencia judicial internacional, litispendencia, garantías procesales.
\end{abstract}

\begin{abstract}
English authorities does not prevent the Spanish courts, the country where the child had his habitual residence before the transfer, assume jurisdiction to declare the wrongfulness of the removal and adopt precautionary measures to avoid a new international abduction.

Keywords: international child abduction, legal kidnapping, return of the child, prompt return, declaration of wrongfulness of the transfer, withdrawal of the passport, international jurisdiction, lis pendens, procedural guarantees.

Sumario: I. Introducción. II. Hechos, alegaciones de las partes y cuestiones controvertidas. III. Exhaustividad, congruencia y motivación en los procesos de declaración de ilicitud del traslado. IV. Litispendencia y competencia judicial internacional en los procesos de retorno y declaración de ilicitud del traslado. V. Valoración.
\end{abstract}

\section{Introducción}

1. En el primer número de 2018 de esta Revista, al hilo de otra sentencia de la Audiencia Provincial de las Islas Baleares, se hacía referencia al crecimiento que está experimentando el problema del 
traslado ilícito de menores. Se apuntaba entonces que, en España, en las últimas décadas, los supuestos se han multiplicado por siete'.

2. El texto del nuevo Auto de la Audiencia Provincial de las Islas Baleares que ahora se comenta, no deja claras algunas circunstancias fácticas de la controversia como, por ejemplo, los vínculos conyugales entre las partes en el momento del litigio, ni los derechos y obligaciones que mantienen con el menor. Tampoco detalla el contenido de lo solicitado en cada uno de los procedimientos que se mencionarán. A continuación, se recogen los hechos relevantes que sí aporta el auto.

\section{Hechos, alegaciones de las partes y cuestiones controvertidas}

3. El Auto de la Audiencia Provincial de las Islas Baleares, de 19 de abril de 2018 resuelve la apelación presentada por doña Valentina frente al Auto un Juzgado de Primera Instancia. Dicho juzgado estimó la demanda de don Enrique, declarando "la ilicitud del traslado del hijo menor de las partes, Lucas, acordando como medida cautelar la retirada del pasaporte del menor en el momento en que entre a España por el aeropuerto de DIRECCION $000^{2}$, a fin de asegurar la prohibición de salida del territorio nacional una vez que se encuentre en la isla como consecuencia de la ejecución de la orden de restitución dictada por las autoridades británicas".

4. En efecto, el 29 de junio de 2017, el menor había sido trasladado de España, donde residía habitualmente desde marzo de 2015, a Londres por doña Valentina, sin el consentimiento de don Enrique. El Auto no hace referencia a las relaciones parentales existentes.

El 26 de septiembre de 2017, un tribunal inglés dictó la orden de restitución del menor. Se deduce de los hechos que, en dicho procedimiento llevado a cabo en Inglaterra, doña Valentina interpuso una declinatoria impugnando la competencia judicial internacional del tribunal. Conforme a lo afirmado por don Enrique, en la decisión inglesa se resolvió también la cuestión de la competencia internacional. La resolución británica no fue recurrida después por doña Valentina.

El 2 de octubre de 2017, como se ha apuntado, se dictó el Auto del Juzgado de Primera instancia de las Islas Baleares en procedimiento de patria potestad declarando ilícito el traslado y adoptando la medida cautelar de la retirada del pasaporte. Cabe deducir de los hechos que doña Valentina alegó al iniciarse este procedimiento que existía un procedimiento pendiente en Inglaterra y que, por lo tanto, el juzgado español no podía conocer hasta que no se resolviera la declinatoria planteada en Inglaterra. No se mencionan en los hechos las fechas de inicio de cada uno de los procedimientos. Como hemos señalado en el párrafo anterior, la sentencia del tribunal inglés resolvió la cuestión de la competencia judicial internacional.

El 19 de octubre de 2017 se dictó un Auto en el que se adoptaron medidas coetáneas. En él, según afirma don Enrique, se declaró probado que el domicilio habitual del menor se encontraba en una concreta dirección en España. En ese mismo Auto, el juez desestimó la excepción relativa a la competencia del juez español que había interpuesto doña Valentina. Este Auto no fue recurrido por doña Valentina.

Aunque los hechos no son del todo nítidos, en lo que respecta a las cuestiones de competencia judicial internacional, se ataca al Auto por haber entrado a conocer el juez español de un asunto relativo a la licitud del traslado del menor estando pendiente la resolución una declinatoria en Inglaterra en un procedimiento de solicitud de restitución del menor. La proximidad temporal de ambos procedimientos dificulta el caso y la falta de mención de las fechas de inicio de los procedimientos complica este análisis.

${ }^{1}$ I. ReIG FABADO, "El traslado ilícito de menores en la unión europea: Retorno vs. Violencia familiar o doméstica", $C D T$, (marzo 2018), Vol. 10, núm. 1, pp. 610-619. A diferencia de lo que ocurría en el pasado, actualmente, más del 70\% de los secuestradores son mujeres que ostentan la custodia. Muchas veces lo hacen huyendo de la violencia. V. A. L. CALVo CARAVACA / J. Carrascosa GonzÁlez, Derecho internacional privado, vol. 2, 18a ed., Comares, Granada, 2018, pp. 489.

Sobre la descripción del fenómeno, causas de su aparición y su crecimiento, y características principales, v. B. GómEz BENGOecheA, Aspectos civiles de la sustracción internacional de menores. Problemas de aplicación del Convenio de La Haya de 25 de octubre de 1980, Dykinson, Madrid, 2002, pp. 19-38.

${ }^{2}$ El Auto omite la localidad. 
Ya en fase de recurso, se ataca la competencia del juez español en la medida en que el juez inglés ha resuelto, finalmente, el asunto.

El 19 de abril de 2018, la Audiencia Provincial de las Islas Baleares dictó el Auto que ahora se comenta.

5. La apelante basó sus pretensiones en varios argumentos. En primer lugar, y haciendo referencia a la vulneración de las garantías procesales, alegó falta de exhaustividad en la resolución. Según la apelante, el juez de primera instancia rechazó toda la actividad probatoria intentada propuesta, y basó su resolución en la ausencia de consentimiento del padre al traslado, sin tener en cuenta los motivos de la estancia del menor el año anterior en el lugar de residencia habitual. El Auto aquí analizado no hace tampoco referencia a esos motivos. La apelante considera, en definitiva, que la existencia de una orden judicial de retorno, consentida y consensuada por ella, no acredita la ilicitud del traslado del menor a Inglaterra, por lo que afirma que existe una falta de motivación en el pronunciamiento.

6. Sin embargo, la parte apelada afirma que doña Valentina había reconocido en la vista tanto el traslado a Inglaterra sin consentimiento del padre, como que el domicilio habitual del niño se encontraba en España desde marzo de 2015, y que esto era resultado de una mudanza voluntaria en busca de trabajo y con vocación de permanencia. Señaló además que no se había rechazado a la apelante la presentación de pruebas relacionadas con el objeto del litigio.

7. También con base en una presunta vulneración de las garantías procesales, se impugnó la decisión por falta de congruencia. De acuerdo con lo expuesto por doña Valentina, no procedía que el juzgado abordase los derechos correspondientes a la patria potestad del padre, sobre el que ya había un procedimiento pendiente en el cual se había impugnado además la competencia del juez inglés. Se entiende que cuando alegó la pendencia del proceso inglés y de la declinatoria, esta todavía no había sido resuelta.

8. Enlaza, por tanto, este motivo con el de la alegación de la falta de competencia del juzgado español, pues -como se ha dicho- existía una declinatoria pendiente de ser resuelta en Inglaterra. Además, finalmente, el tribunal inglés resolvió la cuestión de la restitución, por lo que la apelante considera que la cuestión ya está juzgada. Como se puede apreciar, la apelante entiende que en un primer momento se debió apreciar la excepción de litispendencia y en un momento posterior la de cosa juzgada.

9. Sobre pretendida falta de competencia, don Enrique afirmó que la declinatoria inglesa fue resuelta en la sentencia de 26 de septiembre de 2017, que no fue recurrida. La impugnación de la competencia en España por litispendencia fue resuelta en el Auto en el que se dictaron las medidas coetáneas (y en las que se declaró probado el domicilio habitual del menor), y se desestimó la falta de competencia judicial internacional, auto que tampoco fue impugnado.

\section{Exhaustividad, congruencia y motivación en los procesos de declaración de ilicitud del traslado}

10. La Audiencia Provincial desestimó la alegación referente al rechazo de las pruebas planteadas por dos razones. En primer lugar, porque pudiendo solicitar el recibimiento a prueba en la fase de recurso (al amparo del art. 460.2 $1^{\circ}$ LEC), la apelante no lo hizo. Pero, además, sostiene la Audiencia que el pronunciamiento de instancia estaba basado en pruebas: precisamente el reconocimiento de la madre en la vista de que se llevó al niño sin el consentimiento del padre y su admisión, implícita, de que su llegada a España tenía vocación de permanencia.

11. La Audiencia considera que el auto de primera instancia tampoco incurre en falta de motivación. Recuerda para ello cuál es el objeto del proceso, que es sobre el que se pronunció el juzgado de forma motivada valorando los elementos de prueba relevantes para ello, que son básicamente dos: cuál 
era el domicilio habitual en el momento de la sustracción y la ausencia de consentimiento del padre para que el menor se trasladara a Inglaterra de forma definitiva.

12. En relación con la congruencia del pronunciamiento, el auto señala que las medidas adoptadas por el juzgado son las que prevé el art. 158.3 CC para proteger los derechos de custodia y visitas en el sentido del art. 5 del CH 1980.

13. Según el tribunal de apelación, las cuestiones abordadas no exceden del objeto del procedimiento y de los intereses que deben protegerse en el mismo. El supuesto encaja, según la sala, en una vulneración de los derechos a los que se refiere el Convenio, en la medida en que la conducta de doña Valentina al trasladar al menor a Inglaterra no solo conculca el derecho del padre a intervenir en esa decisión, sino que impide o dificulta a este la posibilidad de ejercer su derecho de custodia o de visitas.

14. En muchos casos la autoridad competente parar dictar la orden de restitución solicita que se obtenga una declaración de la ilicitud del traslado, aquí la apelante parece interpretar que en este caso la orden de restitución ha influido para que las autoridades del país de residencia declaren la ilicitud del traslado.

\section{Litispendencia y competencia judicial internacional en los procesos de retorno y declaración de ilicitud del traslado}

15. El aspecto del Auto sobre el que nos queremos centrar, al igual que hace la Sala, es el relacionado con la competencia judicial internacional. Como se ha señalado, la apelante fundaba su impugnación de competencia en la pendencia de la declinatoria que había planteado en Inglaterra.

16. Aunque, como señalábamos, no es mucha la información que ofrece el Auto de la Sala acerca del procedimiento iniciado en la primera instancia, ni de su fecha de inicio, ni de las alegaciones en hecha en el mismo, cabe deducir cuál era su objeto. Siguiendo el cauce del nuevo art. 778 sexies LEC y del art. $158.3 \mathrm{CC}$, buscaba la declaración de ilicitud del traslado y la adopción de medidas para evitar nuevos traslados. En efecto, la reciente Ley 15/2015, de 2 de julio, de la Jurisdicción Voluntaria, derogó los arts. 1901 a 1909 LEC/1881, e introdujo, entre otros, el art. 778 sexies LEC, que lleva por rúbrica "Declaración de ilicitud de un traslado o retención internacional"’.

17. Este artículo está previsto para los casos en los que un menor con residencia habitual en España sea objeto de un traslado internacional conforme a lo que definen los convenios aplicables. Permite a cualquier interesado, al margen del proceso que se inicie para pedir la restitución internacional, dirigirse a la autoridad judicial española competente para conocer del fondo del asunto con la finalidad de obtener una resolución que declare la ilicitud del traslado. Hace una remisión a los cauces procesales del Título I del Libro IV ${ }^{4}$ para la adopción de medidas definitivas o provisionales en España y las medidas del art. 158 CC.

18. La Fiscalía General del Estado ha elaborado una importante Circular $^{5}$ que viene a completar lo previsto sobre estos procedimientos.

\footnotetext{
${ }^{3}$ Sobre esta nueva regulación, v., entre otros, J. R. LIÉBANA ORTIZ, "El nuevo proceso relativo a la sustracción internacional de menores", Revista electrónica del Departamento de Derecho de la Universidad de La Rioja (REDUR), núm. 13, 2015, pp. 83-109.

${ }^{4}$ Aunque la Ley no es clara, parece que hay que acudir al juicio verbal (J.L. Gómez CoLOMER, "Procesos civiles no dispositivos", en J. Montero Aroca, J.L. Gómez Colomer, S. Barona Vilar, M.P. Calderón Cuadrado, Derecho Jurisdiccional II Proceso Civil, 24a ed., Tirant lo Blanch, Valencia, 2016, p. 780.

${ }^{5}$ Circular 6/2015, sobre aspectos civiles de la sustracción internacional de menores, Anuario de justicia de menores, núm. 15, 2015, pp. 601-694.
} 
19. Es importante señalar en este punto que, aunque no aparece mencionado en el Auto, en los traslados ilícitos intraeuropeos no solo hay que tener en cuenta lo previsto en el CH/1980, sino también, y muy especialmente, lo previsto en el Reglamento Bruselas II bis (en adelante RB II bis) ${ }^{6}$. Como explica Garcimartín, de la aplicación combinada de ambos instrumentos se deriva la existencia de dos vías para hacer frente a un secuestro. Por un lado, el art. 10 del RB II bis prevé la competencia de los tribunales estado miembro en el que residía habitualmente el menor antes del traslado. Esta competencia se mantiene en la medida en que no concurran las circunstancias que enumera ese mismo artículo ${ }^{7}$.

20. La otra vía es solicitar la restitución inmediata ante las autoridades del Estado miembro don-

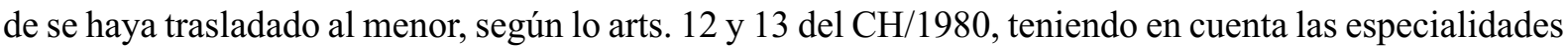
establecidas por el art. 11 del RB II bis ${ }^{8}$. Entre otras cosas, el art. 11 exige a los tribunales de los Estados miembros que se vele por dar audiencia al menor y se resuelva en un plazo máximo de 6 semanas.

21. Es interesante resaltar que el párrafo 8 del art. 11 del RB II bis prevé que con independencia de la resolución dictada en el país en el que se encuentra desplazado el menor, cualquier resolución judicial posterior que ordene la restitución del menor, dictada por un órgano jurisdiccional de la residencia habitual anterior al traslado será ejecutiva con el fin de garantizar la restitución del menor.

22. Es cierto que no en el caso en estudio, la resolución española no ordena la restitución, sino que declara el traslado ilícito y ordena medidas cautelares, sin embargo, puede deducirse que no cabe oponerse a la competencia del juez de la residencia alegando que el juez del lugar de traslado a resolvió la cuestión.

23. El art. 778 sexies LEC contiene dos párrafos claramente diferenciados. Ambos hacen referencia a solicitudes de declaración de ilicitud de un traslado. Sin embargo, mientras que el primero alude a solicitudes al margen del procedimiento que se inicie para solicitar la restitución internacional, y que muchas veces tendrán como objetivo la adopción de medidas definitivas o provisionales en España, en especial las previstas en el art. $158 \mathrm{CC}$ (como ocurre en el caso aquí analizado). Por su parte, el segundo estaría específicamente redactado para desarrollar al art. 15 del CH.1980, es decir, para casos en los que las autoridades del país donde se ha producido el traslado requieran al solicitante una resolución de las autoridades de la residencia. Por otro lado, mientras que para la declaración a la que ser refiere el párrafo primero, la competencia corresponde a la autoridad competente para conocer sobre el fondo del asunto, en el segundo se establece que la competencia corresponde, en principio, a la última autoridad judicial que haya conocido en España de cualquier proceso sobre responsabilidad parental sobre el menor, y en su defecto, el juzgado de primera instancia del último domicilio del menor en España.

${ }^{6}$ V. entre otros, P. JimÉnEz Blanco, Litigios sobre la custodia y sustracción internacional de menores, Marcial Pons, 2008, pp. 169-207.

${ }^{7}$ Los tribunales de la residencia anterior al traslado "conservarán su competencia hasta que el menor haya adquirido una residencia habitual en otro Estado miembro y:

a) toda persona, institución u organismo que tenga el derecho de custodia haya dado su conformidad al traslado o a la retención,

o bien

b) el menor, habiendo residido en ese otro Estado miembro durante un período mínimo de un año desde que la persona, institución u organismo que tenga el derecho de custodia haya tenido o hubiera debido tener conocimiento del paradero del menor, esté integrado en su nuevo entorno y se cumpla alguna de las condiciones siguientes:

i) que en el plazo de un año desde que el titular del derecho de custodia haya tenido o hubiera debido tener conocimiento del paradero del menor, no se haya presentado ninguna demanda de restitución ante las autoridades competentes del Estado miembro al que se haya trasladado o en el que esté retenido el menor,

ii) que se haya desistido de una demanda de restitución presentada por el titular del derecho de custodia sin que haya presentado ninguna nueva demanda en el plazo estipulado en el inciso i),

iii) que se haya archivado, a tenor de lo dispuesto en el apartado 7 del artículo 11, una demanda presentada ante un órgano jurisdiccional del Estado miembro en el que el menor tenía su residencia habitual inmediatamente antes de su traslado o retención ilícitos,

iv) que los órganos jurisdiccionales del Estado miembro en el que el menor tenía su residencia habitual inmediatamente antes de su traslado o retención ilícitos hayan dictado una resolución sobre la custodia que no implique la restitución del menor".

${ }^{8}$ F. Garcimartín Alférez, Derecho internacional privado, Civitas, 2017, p. 160. 
24. Como apuntó la Audiencia, no cabe hablar aquí de litispendencia, dado que el objeto del litigio inglés es distinto -solicitud de retorno del menor-. En el presente se trata solo de aclarar si un menor con residencia en España ha sido objeto de un traslado o retención transnacional conforme a lo establecido en el Convenio. De no ser así, como señala la Audiencia Provincial, "se pondría en riesgo la propia celeridad y especifidad de unas actuaciones judiciales que pretenden evitar la sustracción de los hijos, como es el objeto de las medidas previstas en el art. 158.3 CC". Como afirma el auto de la sala, este tipo de medidas pueden adoptarse en cualquier procedimiento civil o penal, incluso en un expediente de jurisdicción voluntaria.

25. A lo largo del articulado del CH 1980 se observa que la intención de la norma es evitar que decisión de las autoridades del país donde el menor ha sido desplazado interfiera en los procesos que se lleven a cabo en el país de residencia. En este sentido, el art. 19 del Convenio aclara que "una decisión adoptada en el marco del presente Convenio sobre la restitución del menor no afectará a la cuestión de fondo del derecho de custodia". Por tanto, está prevista la plena compatibilidad de pronunciamientos, ya que los que se siguen al amparo del Convenio, buscan solamente una acción directa que permita el retorno a la situación de hecho anterior al traslado del menor. Por esta razón, el CH 1980 suele calificarse como de un "convenio fáctico".

26. Es más, uno de los objetivos del Convenio es evitar que los tribunales del país donde se ha trasladado ilícitamente al menor se declaren competentes para conocer de los derechos relacionados con la responsabilidad parental (cosa que con frecuencia busca el progenitor que ha perpetrado la sustracción). En este sentido, el art. 16 del $\mathrm{CH} / 1980$ prohíbe a los tribunales del país al que ha sido trasladado ilícitamente el menor conocer sobre el fondo de los derechos de custodia salvo que se determine que el menor no tiene que ser restituido o que haya transcurrido un período de tiempo razonable sin que se presente la solicitud. Se trata en cierto modo de una regla de "competencia judicial internacional negativa"

27. Por otro lado, el CH 1980 prevé que se puedan dictar decisiones relativas a la custodia del menor, sin que ello deba afectar a la decisión sobre la restitución, aunque puedan tenerse en cuenta los motivos de dicha decisión al aplicar el convenio (art. 17).

28. Hay que recordar que el objetivo del CH 1980 es, primordialmente, otorgar la restitución inmediata del menor al país de su residencia habitual. "Cuando un tribunal se pronuncia sobre la restitución del menor, en ningún caso se está pronunciando sobre la custodia del menor"11.

29. Como apuntan Calvo y Carrascosa, "en el caso de sustracción ilícita de un menor desde España a otro país, los tribunales españoles estiman que, cuando deben decidir sobre la custodia del menor, la conducta del progenitor sustractor constituye un dato a tener presente para modificar, en su perjuicio, la custodia del menor, pues el hecho del traslado y del incumplimiento del mandato judicial permite afirmar un ejercicio inadecuado de la guarda de su hija por la madre y la incapacidad de esta para ejercer una guarda responsable de la misma (AAP Málaga 15 mayo 2017). El objetivo es que sean los tribunales del lugar donde el menor tiene su residencia habitual los que se pronuncien sobre la guarda y custodia y visitas del menor.

30. Como señalan estos mismos autores, el objetivo del CH 1980 es volver al statu quo anterior y evitar que se consolide la situación creada por el secuestro, nunca entrar al fondo del asunto. El CH 1980 parte de que las autoridades de la residencia habitual anterior son las mejor situadas para decidir

9 A. L. Calvo Caravaca / J. Carrascosa González, Derecho internacional privado, vol. 2, $18^{\mathrm{a}}$ ed., Comares, Granada, 2018, pp. 494.

${ }^{10}$ A. L. Calvo Caravaca / J. Carrascosa GonzÁlez, Derecho internacional privado, vol. 2, $18^{\mathrm{a}}$ ed., Comares, Granada, 2018, pp. 495.

${ }^{11}$ A. L. Calvo Caravaca / J. Carrascosa GonzÁlez, Derecho internacional privado, vol. 2, $18^{\text {a }}$ ed., Comares, Granada, 2018, pp. 495 y 503, citando el AAP Barcelona 13 marzo 2012 y la SAP Las Palmas 29 junio 2017. 
sobre la custodia del menor, tanto por su más fácil acceso a la información sobre el menor como por su mejor posición para aplicar cualquier medida. ${ }^{12}$

\section{Valoración final}

31. La problemática suscitada en este proceso puede deberse a la celeridad con que con frecuencia actúan los tribunales ingleses a la hora de dictar órdenes de retorno. Al adelantarse al pronunciamiento sobre la declaración de ilicitud en España, se han planteado dudas sobre la existencia de una situación de litispendencia o, incluso, de cosa juzgada. Sin embargo, la Audiencia acierta con su solución que permite evitar tácticas dilatorias.

32. Pese a que del texto del Auto no se deducen todos los datos relevantes para conocer el contenido del proceso en España y de su objeto, es previsible que en casos como el aquí analizado, puedan repetirse alegaciones relacionadas con la competencia judicial internacional cuando se inicien procesos en los distintos países implicados. Convendría aclarar esta cuestión para que la aplicación del art. 778 sexies LEC no vuelva a generar cuestiones como las aquí planteadas.

33. La concurrencia de normas nacionales e internacionales en este tipo de procesos, así como la intervención de autoridades de distinta naturaleza y de distintos países añade dificultad a la correcta solución de una problemática tan delicada y, por desgracia, tan frecuente como es la sustracción internacional de menores. Convendría seguir estableciendo guías claras (como se ha hecho desde la fiscalía) y continuar intensificando la formación en este sector de todos los operadores implicados.

${ }^{12}$ A. L. Calvo Caravaca / J. Carrascosa González, Derecho internacional privado, vol. 2, $18^{\text {a }}$ ed., Comares, Granada, 2018, pp. 495-496. 